\title{
Stone Soup
}

\section{Acronyms and Abbreviations Used in the Ocean Drilling Program}

Ocean Drilling Program

Texas A\&M University

Technical Note No. 13

Compiled by Elizabeth A. Heise

Ocean Drilling Program

Texas A\&M University

College Station, Texas 77845-9547

U.S.A.

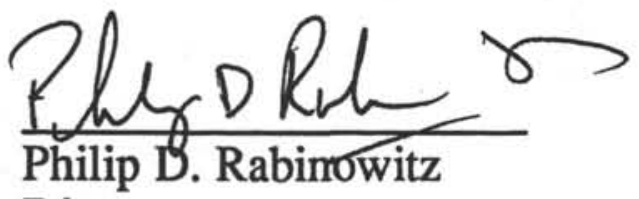

Director

Tor Trancis

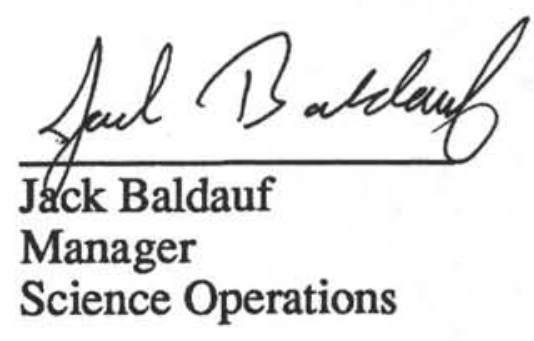

Timothy J.G. Francis

Deputy Director 
Material in this publication may be copied without restraint for library, abstract service, educational or personal research purposes; however, republication of any portion requires the written consent of the Director, Ocean Drilling Program, Texas A\&M University Research Park, 1000 Discovery Drive, College Station, Texas 77845-9547, U.S.A., as well as appropriate acknowledgment of this source.

\author{
Technical Note No. 13 \\ First Printing 1993 \\ Distribution
}

Copies of this publication may be obtained from the Director, Ocean Drilling Program, Texas A\&M University Research Park, 1000 Discovery Drive, College Station, Texas 77845-9547, U.S.A. In some cases, orders for copies may require a payment for postage and handling.

\title{
DISCLAIMER
}

This publication was prepared by the Ocean Drilling Program, Texas A\&M University, as an account of work performed under the international Ocean Drilling Program, which is managed by Joint Oceanographic Institutions, Inc., under contract with the National Science Foundation of the United States. Funding for the program is provided by the following agencies:

Canada/Australia Consortium for the Ocean Drilling Program

Deutsche Forschungsgemeinschaft (Federal Republic of Germany)

Institut Français de Recherche pour l'Exploitation de la Mer (France)

Ocean Research Institute of the University of Tokyo (Japan)

National Science Foundation (United States)

Natural Environment Research Council (United Kingdom)

European Science Foundation Consortium for the Ocean Drilling Program

(Belgium, Denmark, Finland, Iceland, Italy, Greece, The Netherlands,

Norway, Spain, Sweden, Switzerland, and Turkey)

Academy of Sciences (Russia) - (Inactive)

Any opinions, findings, and conclusions or recommendations expressed in this publication are those of the author(s) and do not necessarily reflect the views of the National Science Foundation, the participating agencies, Joint Oceanographic Institutions, Inc., Texas A\&M University, or Texas A\&M Research Foundation. 


\section{INTRODUCTION}

This Technical Note has been developed to facilitate communication between representatives from various disciplines. The list presented in the following pages represents a compilation of acronyms and abbreviations commonly used by the Ocean Drilling Program/Joint Oceanographic Institutions for Deep Earth Sampling (ODP/JOIDES) personnel and incorporates terminology relevant to science and engineering, operations, computers services, publishing, and curation of the Ocean Drilling Program.

\section{STONE SOUP}

A\&G-DPG [JOIDES] Atolls and Guyots Detailed Planning Group

AABW [science] Antarctic Bottom Water AAPG American Association of Petroleum Geologists

AAS [science] atomic-absorption spectroscopy

AASP American Association of Stratigraphic Palynologists

ABM [publications] assigned board member (of the ERB)

ABS [logistics] American Bureau of Shipping

ACAD computer-aided drafting (a.k.a. AUTOCAD, CAD, CADD)

ACT [logging] neutron porosity (Cf source) logging tool (aluminum clay tool)

AD [drilling] assistant driller

A/D [science] Analog to Digital Converter

AEG Association of Engineering Geologists

AF [science] alternating field (demagnetization); [logistics] air freight

AGI American Geological Institute

AGU American Geophysical Union

AMS [logging] auxiliary measuring sonde (a Schlumberger tool that can be added to any digital string to give temperature and tension measurements); [science] anisotropy of magnetic susceptibility

APC [drilling] advanced hydraulic piston corer

API American Petroleum Institute; [computers] application programming interface

ARM [science] anhysteretic remanent magnetization

ARP [JOIDES] Atlantic Regional Panel
ASCII [computers] American Standard Code for Information Interchange

ASK [ship] automatic station keeping (see DPS)

atm [science] atmospheres

AWB [logistics] airway bill for air-freight shipments

AWG Association for Women Geoscientists

AZ [science] acme zone (biostratigraphy)

B.A.S. British Antarctic Survey

bbl [drilling] barrel (unit of measure)

BBS [computers] bulletin-board system

bec blind courtesy copy

BCOM [JOIDES] Budget Committee

BGDS [drilling] bit-guide deployment system

BGR Bundesanstalt für Geowissenschaften und Rohstoffe (Federal Republic of Germany)

BGS British Geological Survey

BHA [drilling] bottom-hole assembly

BHC [logging] borehole compensated sonic logging tool

BHP [logging] bottom hole pressure

BHT [logging] bottom hole temperature

BHTV [logging] borehole televiewer downhole tool

BHV [logging] Baker Hughes Video

BIOS [computers]basic input/output system

B/L [logistics] bill of lading for surface (ocean) freight

BLAST [computer/communications] BLocked ASynchronous Transmission; protocol used for PMAIL or telexes over Marisat

BMR Bureau of Mineral Resources (Australia) 
Technical Note No. 13

Page 4

bomb [science/drilling] pressure-measuring device or sample container

BOMB [curation] carbonate analysis (sample designation mostly defunct)

BOP [drilling] blowout preventer

BOS [drilling] back-off sub

BP British Petroleum

BPH [drilling] breakaway piston head (for APC)

brf [drilling] below rig floor (as in mbrf: meters below rig floor, by drill-pipe measurement)

BRG Borehole Research Group, LamontDoherty Earth Observatory

BRGM Bureau de Recherches Géologiques et Minières (France)

BSE [science] back-scattered electron

bsf [science] below seafloor (as in mbsf: meters below seafloor)

bsl [science] below sea level (as in mbsl: meters below sea level; corrected--using ship's draft-from drill-pipe measurements

BSR [science] bottom-simulating reflector

BT [science] bathythermograph (as in XBT: expendable bathythermograph)

btu [science] British thermal unit

BVA [drilling] ball-valve assembly

BW [drilling] borehole water

$\mathbf{C}_{\text {org }}$ [science] organic carbon

$\mathbf{C}_{1} / \mathbf{C}_{2}$ [science] methane/ethane ratio

$\mathbf{C}_{6+}$ [science] hexane and higher hydrocarbons

CAD computer-aided design and drafting (a.k.a. AUTOCAD, ACAD, CADD)

CADA [drilling] cam-actuated drill-ahead; feature of DQ running tool system

CALI [logging] caliper logging tool

CAP-DPG [JOIDES] Cascadia Accretionary Prism Detailed Planning Group

CASSI Chemical Abstracts Service Source Index

CB [drilling] center bit

cc carbon copy (anachronistic term!)

CCD [science] carbonate compensation depth

CD-ROM [computers] compact disc--read-only memory

CDS [drilling] colleted delivery system

CEPAC [JOIDES] Central and Eastern Pacific Panel

CEPDPG [JOIDES] Central and Eastern Pacific Detailed Planning Group

CFP- Compagnie Française du Pétrole (France) cgs [science] centimeter-gram-second system of units

CHEMDB [ODP] database program for carbonate, interstitial water, gas chromatography, and Rock-Eval analysis results

CHL [drilling] core height logger (electronics unit of SCM system)

CHN [science] carbon-hydrogen-nitrogen analyzer

CMD [drilling] cable measuring device

CNRS Centre Nationale de Recherche Scientifique (France)

CNS [science] carbon-nitrogen-sulfur analyzer

CNT-G [logging] thermal and epithermal neutron porosity ( $\mathrm{Am} / \mathrm{Be}$ source) logging tool (Schlumberger version G)

CORELOG [ODP] database program for coreinventory information

CORK [drilling] reentry cone seal and instrument feed thru

COSOD Conference on Scientific Ocean Drilling

COST [science] Continental Offshore Stratigraphic Test

CPA [ship] closest point of approach, in nmi cps [science] counts per second or cycles per second

CPI [science] carbon preference index

CPU [computers] central processing unit

CRC [science] concurrent range zone (biostratigraphy)

CRM [science] chemical remanent magnetization

CSDP [JOIDES] Continental Shelf Drilling Program

CSES [drilling] conical side-entry sub

CSG [ODP] Computer Services Group (obsolete)

CSU [logging] cyber service unit

CT [drilling] coring technician

CY calendar year

D5BC [ship] radio call sign of SEDCOIBP 471

DAC [computers] digital-to-analog converter

DARPA Defense Advanced Research Projects Agency (U.S. Navy)

DBMI [drilling] drill-bit motion indicator

DC [drilling] drill collar, [electronics] direct current

DCB [drilling] diamond core barrel 
DCE [computers] distributed computing environment

DCS [drilling] diamond coring system

DDE [computers] dynamic data exchange

DEC [computers] Digital Equipment Corporation

DES [drilling] dual-elevator stool on the rig floor (the top of the DES is the point from which drill-pipe measurements are made)

DFD [science] data-file documents

DI-BHA [drilling] drill-in bottom-hole assembly

DIC [drilling] drill-in casing

DIL [logging] dual induction log (resistivity)

DITE [logging] digital dual induction tool (resistivity), a.k.a. phasor induction tool

DLL [logging] dual laterolog (resistivity)

DMI [computers] desktop-management interface

DML [logging] downhole measurements laboratory aboard JOIDES Resolution (a.k.a. DHML and DHL)

DMP [JOIDES] Downhole Measurements Panel

DOS [computers] Disk Operating System

DOSECC Deep Observation and Sampling of the Earth's Continental Crust

DP [ship] dynamic positioning; [drilling] drill pipe; [computer] data processing

DPG [JOIDES] Detailed Planning Group

DPM [drilling] drill-pipe measurement

DPO [ship] dynamic positioning operator

DPS [ship] dynamic positioning system

DQ [drilling] Dril-Quip, manufacturer of casing running tools

DR [ship] dead reckoning

DRB [drilling] DCS retractable bit system

DRM [science] depositional remanent magnetization

DS [drilling] drilling superintendent

DSDP Deep Sea Drilling Project

DSRV [science] deep submersible research vessel

DSS [drilling] deep sound (sonic) source, 3.5$\mathrm{kHz}$ bottom profiler deployed on drill string

DST [drilling] drill-stem test

DTD [computers] document-type definition

DW [drilling] draw works

ECOD European Science Foundation (ESF) Consortium for the Ocean Drilling Program
ECR [ODP] East Coast Repository at LDEO; [ship] ship's engine-control room

EDI [computers] electronic data interchange

EDO EDO-Western, manufacturer of reentry sonar system and seismic recorders

EDS [science] energy dispersive spectrometry

EEZ Exclusive Economic Zone

EI [computers] electronic imaging

EIA [computers] enterprise information architecture

e-mail [computers] electronic mail

EMCO ESF Management Committee for the ODP

EMD [ship] electromotive diesel (engines and electric motors)

EMR Department of Energy, Mines and Resources (Canada)

EOM [science] extractable organic matter

EMS [drilling] electronic multishot instrument

EPR [science] East Pacific Rise

EPR-DPG [JOIDES] East Pacific Rise Detailed Planning Group

EPS [computers] encapsulated PostScript

ERB [publications] Editorial Review Board

ESCO ESF Scientific Committee for ODP

ESF European Science Foundation (in terms of ODP, "the European Science Foundation Consortium for the Ocean Drilling Program": Belgium, Denmark, Finland, Greece, Iceland, Italy, The Netherlands, Norway, Spain, Sweden, Switzerland, and Turkey)

ET [ODP] electronics technician

ETA estimated time of arrival

ETD estimated time of departure

ETH Eidgenössische Technische Hochschule (Switzerland)

EX [science] extinction

EXCOM [JOIDES] Executive Committee

FA or FAD [science] first-appearance datum (biostratigraphy)

FAMOUS [science] Franco-American MidOcean Undersea Study

FCO [science] first common occurrence (biostratigraphy)

FCSP [curation] final cruise sampling program (end-of-cruise summary of each sample request for a leg; included in Hole Summary) 
Technical Note No. 13

Page 6

FEA finite-element (stress) analysis

FFF [drilling] free-fall funnel (a.k.a. minicone)

FH [drilling] full hole

FID [science] flame ionization detector (gas chromatography)

FMS [logging] Formation Microscanner logging tool

FO [science] first occurrence (biostratigraphy)

FPAPWG [JOIDES] Fluid Processes in Accretionary Prisms Working Group

FRG Federal Republic of Germany

FTP [computers] file-transfer protocol

FUBAR fouled up beyond all recognition

FY fiscal year

FYF for your files

FYI for your information

FZ [science] fracture zone

GC [science] gas chromatograph; [DSDP] GLOMAR Challenger

GCR [ODP] Gulf Coast Repository at TAMU

GEBCO General Bathymetric Chart of the Oceans

GEOMAR F.R.G. Research Center for Marine Geosciences

GEOPROPS geophysical properties tool

GEOREF geological reference database of the American Geological Institute

GER [curation] Geriatric Core Study

GIS Geoscience Information Society; geographic information system(s)

GMT Greenwich Mean Time (replaced by UTC)

go-devil [logging] a tool that free-falls down the drill pipe

GPIT [logging] three-axis magnetometerinclinometer logging tool (also G-PIT)

gpm [drilling] gallons per minute (pump rate)

GPS [ship] Global Positioning System (satellite navigation system)

GQL [computers] graphical query language

GR [logging] natural gamma-ray logging tool

GRAPE [science] gamma-ray attenuation porosity evaluator (bulk-density analyzer)

GS [drilling] overshot for wireline coring

GSA Geological Society of America

GSC Geological Survey of Canada

GSGP [JOIDES] Global Sedimentary Geology Program

GST [logging] induced gamma-ray spectroscopy logging tool
HARVI [ODP] database program for visual descriptions of hard-rock cores

HBR [drilling] hydraulic bit release (obsolete)

HC [drilling] heave compensator; [science] hydrocarbons

HEE [drilling] high-energy engineering (pipesevering system)

HF [science] heat flow; high frequency; hydrofluoric acid

HFSE [science] high-field-strength elements

HFU heat-flow units

HI [science] hydrogen index

HIG Hawaii Institute of Geophysics (University of Hawaii)

HLDT [logging] slim-hole lithodensity logging tool

HP Hewlett-Packard

HPC [drilling] hydraulic piston corer (replaced by the advanced hydraulic piston corer, APC)

HRB [drilling] hard-rock guide base

HREE [science] heavy rare-earth element(s)

HRGB [drilling] hard-rock guide base

HRO [drilling] hard-rock orientation

HRTHIN [ODP] database program for hardrock thin-section descriptions

HS [drilling] head sub

HVDYN [drilling] heave dynamics drill-string program

HWDJ [drilling] heavy-wall drilling joint

IADC International Association of Drilling Contractors

IAPSO International Association for the Physical Sciences of the Oceans (provides bottled standard seawater for analytical calibrations)

IBM International Business Machines (used generically as a term to describe personal computers)

ICP [science] inductively coupled plasma spectroscopy

ID inside diameter

IDAS isothermal decompression analysis system

IDSS [drilling] instrumented drill-string sub

IDW [drilling] integrated depth wheel; [science] Indian Deep Water

IFP Institut Français du Pétrole (France)

IFREMER Institut Français de Recherche pour l'Exploitation de la Mer (France) 
IGC International Geological Congress IHP [JOIDES] Information Handling Panel

IID [logging] deep induction resistivity ILM [logging] medium induction resistivity ILP [JOIDES] International Lithosphere Program

ILW [science] International Low Water

INAA [science] instrumental neutron activation analysis

IOC Intergovernmental Oceanographic Commission

IOP [JOIDES] Indian Ocean Panel

IPG Institut de Physique du Globe (France)

IPOD International Phase of Ocean Drilling

IR [drilling] iron roughneck; [science] infrared; insoluble residue; [publications] Initial Reports of the Proceedings of ODP

IRM [science] isothermal remanent magnetization

IS [computers] information system(s)

ISD [ODP] Information Services Department

ISS [science] ion-scattering spectrometry

IT [computers] information technology

IW [curation] interstitial water (sample designation)

JOI Joint Oceanographic Institutions, Inc.

JOI-BOG [JOI] JOI Board of Governors

JOIDES Joint Oceanographic Institutions for Deep Earth Sampling

JPL Jet Propulsion Laboratory

JD Julian Day

KB [drilling] kelly bushing

KFZ [science] Kane Fracture Zone

K/T [science] Cretaceous/Tertiary boundary

KTB German continental deep-drilling project

KWG [JOIDES] Kerguelen Working Group

LA or LAD [science] last-appearance datum (biostratigraphy)

LAN [computers] local-area network

LANL Los Alamos National Laboratory

LAS [science] laboratory automation system

LAST [logging] lateral-stress tool

LBL Lawrence Berkeley Laboratory

LCD [computers] liquid crystal display

LD [drilling] lay down

LDEO Lamont-Doherty Earth Observatory (formerly LDGO)

LDGO Lamont-Doherty Geological Observatory (now LDEO)

LDT [logging] lithodensity logging tool
LEGS [ODP] database program to write reports with leg, site, hole, and core-recovery information

LEL [safety] lower expansive limit

LFV [drilling] lockable float valve

LH [drilling] left hand

LILE [science] large ion lithophile element

LITHP [JOIDES] Lithosphere Panel

LLD [logging] deep laterolog

LLS [logging] shallow laterolog

LO [ODP] laboratory officer, [science] last occurrence (biostratigraphy)

LOI loss on ignition

LREE [science] light rare-earth element(s)

LRP [JOIDES] long range plan

LSB [drilling] lower support bearing

LSS [logging] long-spaced sonic logging tool

$\mathrm{Ma}$ [science] million years (ago)

Mac [computers] Apple Macintosh computer

MAR [science] Mid-Atlantic Ridge

MARK [science] Mid-Atlantic Ridge Kane Fracture Zone

MATMAN [logistics] ODP (materials management) inventory database system

MAXIS [logging] multi-tasking acquisition and imaging system

MBR [drilling] mechanical bit release

mbrf [drilling] meters below rig floor

mbsf [science] meters below seafloor

mbsl [science] meters below sea level

MCD [logging] caliper tool, measures hole diameter

MCS [science] multichannel seismic; [logging] multichannel sonic (12-channel) logging tool

MCT [drilling] motor control transformer

M-D [drilling] Martin-Decker rig-weight indicator

MDCB [drilling] motor-driven core barrel (replaces NCB)

MHS [computers] message-handling system

MIB [computers] management information base

MIS [computers] management information system(s)

ML [logging] microlog

MODU Mobile Offshore Drilling Unit

Moho [science] Mohorovicic discontinuity

MOM [ship] SEDCO's Marine Operations Manual

MORB [science] mid-ocean-ridge basalt

MOU Memorandum of Understanding 
Technical Note No. 13

Page 8

MRT [science] maximum-reading thermometer MSA Mineralogical Society of America

MSDS [logistics] Marine Science and Development Shop (SIO); [OSHA] Material Safety Data Sheet

MSL mean sea level

MSP [curation] master sampling plan (defunct term, replaced by FCSP)

MST [science] multisensor track

MT [ODP] marine technician; eMpTy

MU [drilling] make up

MWC [drilling] measurement while coring

MWD [drilling] measurement while drilling

m.y. [science] million years

NAAG-DPG [JOIDES] North Atlantic-Arctic Gateways Detailed Planning Group

NADW [science] North Atlantic Deep Water

NAMOC [science] Northwest Atlantic MidOcean Canyon

NARM-DPG [JOIDES] North Atlantic Rifted Margins Detailed Planning Group

NCB [drilling] Navidrill core barrel (replaced by $\mathrm{MDCB}$ )

NERC Natural Environment Research Council (U.K.)

NFG not functioning good: useless

NFS [computers] network file system

NGA [science] natural gas analyzer

NGDC National Geophysical Data Center (U.S.)

NGT [logging] natural-gamma spectrometry logging tool

NMDL [drilling] non-magnetic (stainless steel) drill collar

nmi [science and boating] nautical mile

NRC [U.S. Govt.] Nuclear Regulatory Commission

NRM [science] natural remanent magnetization

NSB National Science Board (U.S.)

NSF National Science Foundation (U.S.)

NSO [science] nitrogen, sulfur, oxygen

NSOW [science] Norwegian Sea Overflow Water

OBS [science] ocean-bottom seismometer

OCB [drilling] outer core barrel

OCR [computers] optical character recognition

OCS [science] Outer Continental Shelf

OD outside diameter

ODL Overseas Drilling Limited

ODP Ocean Drilling Program
ODPC ODP Council

ODWG [JOIDES] Offset Drilling Working Group

OG [curation] organic geochemistry (sample designation)

OHP [JOIDES] Ocean History Panel

OI [science] oxygen index

OLE [computers] object linking and embedding

OLTP [computers] on-line transaction processing

$\mathbf{O M}$ [science] organic matter

OMDP [JOIDES] Ocean Margin Drilling Program

ONDO [ODP] ODP Nankai Downhole Observatory

ONR [U.S. Govt.] Office of Naval Research

OOP [computers] object-oriented programming

ORI Ocean Research Institute, University of Tokyo (Japan)

OS [drilling] operations superintendent; [computers] operating system

OSE [logging] oblique seismic experiment

OSHA U.S. Occupational Safety and Health Administration (not just a small town in upstate Wisconsin)

OSN Ocean Seismic Network

OSU Oregon State University (rarely, Ohio State University; hardly ever, Oklahoma State University)

OT [computers] object technology

OTC Offshore Technology Conference

PC photocopy; [computers] personal computer (as in IBM PC), program counter; [electronics] printed circuit

PCB [drilling] pressure core barrel (obsolete DSDP tool)

PCD [drilling] polycrystalline diamond

PCOM [JOIDES] Planning Committee

PCS [drilling] pressure core sampler

PCSP [curation] preliminary cruise sampling plan (brief outline of each sample request accepted, rejected, or deferred at the beginning of each leg)

PDB [science] Peedee belemnite, a standard for oxygen- and carbon-isotope analyses

PDC [drilling] polycrystalline diamond compact drill bit

PDCM [drilling] positive displacement coring motor 
PDM [drilling] positive displacement (drilling) motor

PDR [science] precision depth recorder; [ODP] Philip D. Rabinowitz

PDRM [science] post-depositional remanent magnetization

PEC [JOIDES] Performance Evaluation Committee

PERC [publications] Preliminary Editorial Review Check

PI [ODP] principal investigator

PIE [computers] personal interactive electronics

PIG [drilling] drill-pipe internal rust scraper

PMS [ship] power-management system; preventive-maintenance system

POOH [drilling] pull out of hole (in reality, lift the bit up off the bottom of the hole, not necessarily clear the mud line)

PP [science] physical properties (a.k.a. fizz props)

ppb [science] parts per billion

ppm [science] parts per million

PPSP [JOIDES] Pollution Prevention and Safety Panel (a.k.a. Safety Panel)

PRISM [computers] projection and integrated stand-alone monitor

PRO [computers] Digital Equipment Corporation PRO-350 or PRO-380 computer (obsolete)

psi [science] pounds per square inch

$\mathbf{P} / \mathbf{U}$ [drilling] pick up

PVC [logistics] polyvinyl chloride

$\boldsymbol{P}$-wave [science] primary seismic or compressional wave

PWS [science] pore-water sampler

QC quality control

Quad combo [logging] string of logging tools including dual induction, litho-density, neutron, natural gamma ray, sonic

RAM [computers] random-access memory

RBI [drilling] Rock Bit International (drill bit manufacturer)

RCB [drilling] rotary core barrel

$\mathbf{R} / \mathbf{D}$ [drilling] rig down

RDX [drilling] explosive used in pipe-severing system

$\mathbf{R} / \mathbf{E}$ [drilling] reentry

REE [science] rare-earth element(s)

reefer [curation] refrigerated core/sample storage area
REPSAM [curation] repository sampling computer program

RFP [ODP] request for proposals

RFQ [ODP] request for quotes

RFT [drilling] retrievable formation tester (water sampler)

RIH [drilling] run in hole (actually, lower drill string below the rig floor, not necessarily below the mud line)

RISC [computers] reduced instruction set computing

RKB [drilling] rotary kelly bushing

RMS [science] root mean square (e.g., in seismic velocities)

Rocky [ODP] Data entry program for hand specimen and thin section description of hard rocks

ROM [computers] read-only memory

ROP [drilling] rate of penetration in drilling or coring

ROV [science] remotely-operated vehicle

rpm [drilling] revolutions per minute

RSMAS Rosenstiel School of Marine and Atmospheric Science (University of Miami)

RST [drilling] rotary shifting tool

RSWG [JOIDES] Red Sea Working Group

RT [drilling] round trip of the drill string (= $\mathrm{POOH}+$ bit change $+\mathrm{RIH})$

RU [drilling] rig up

$\mathbf{R Z}$ [science] range zone (biostratigraphy)

SAM [curation] shipboard sampling computer program

SAMUTL [ODP] curation program to edit sample databases and write sample reports

S/B [drilling] set back

SBA [curation] shipboard analysis (sample designation)

SBDC [drilling] seal bore drill collar

SCM [drilling] sonic core monitor

SCR [electronics] silicon control rectifier

SCS [science] single-channel seismic

SCSI [computers] small computer system interface

SCBA [safety] Self-contained Breathing Apparatus

SCUBA [safety] Self-contained Underwater Breathing Apparatus

SDP [curation] Sample Distribution Policy

SDT [logging] sonic digital logging tool 
SECTIONLOG [ODP] curatorial database for individual core-section histories

SEDCO/BP 471 [ship] registered name of drilling vessel used by ODP; a.k.a. JOIDES Resolution

SEG Society of Exploration Geophysicists

SEM [science] scanning electron microscope

SEM/EDA [science] scanning electron microscope energy dispersive analysis

SEPM Society of Economic Paleontologists and Mineralogists (now called Society for Sedimentary Geology)

SES [drilling] sidewall-entry sub (inserted in the drill string when logging)

SF [logistics] surface freight; [ship] satellite fix

SFL [logging] spherically focused resistivity measurement

SGML [computers] Standard Generalized Markup Language

SGPP [JOIDES] Sedimentary and Geochemical Processes Panel

SI [science] Système International (international system of units)

SID [curation] sample investigations computer database

SIO Scripps Institution of Oceanography (University of California, San Diego)

SLIDES [ODP] database program for smearslide descriptions

SLM [drilling] Steel Line Measurement (measurement of drill string and BHA with steel measuring tape)

SLWG [JOIDES] Sea Level Working Group

SMDC [drilling] short monel drill collar (nonmagnetic)

SMF [computers] standard message format

SMOOTH [ODP] shipboard navigationplotting program

SMOW [science] standard mean ocean water

SMP [JOIDES] Shipboard Measurements Panel

SMS [computers] storage-management system

SN [ship] satellite navigation

$\mathrm{S} / \mathrm{N}$ signal-to-noise ratio

SOE [JOIDES] Special Operating Expense

SOEST School of Ocean and Earth Science and Technology (University of Hawaii)

SOHP [JOIDES] Sediments and Ocean History Panel

SOP [JOIDES] Southern Oceans Panel; [general] standard operating procedure
SOW [JOIDES] Statement of Work

SP [science] shot point (on seismic survey lines); [logging] spontaneous potential

SPE Society of Petroleum Engineers spm [drilling] strokes per minute

SPR [computers] Software Problem Report

SR [publications] Scientific Results of the Proceedings of ODP

SR-DPG [JOIDES] Sedimented Ridges Detailed Planning Group

SSA Seismological Society of America

SSDB [JOIDES] Site Survey Data Bank (at LDEO)

SSP [JOIDES] Site Survey Panel

SSR [drilling] subsea release cementing system

SST [science] sea-surface temperature

SW [drilling] sea (salt) water

$S$-wave [science] secondary wave

TAG [science] Trans-Atlantic Geotraverse

TAI [science] thermal-alteration index

TAMRF Texas A\&M Research Foundation

TAMU Texas A\&M University

tbg [drilling] tubing

TCCB [logging] digital telemetry tool

TCD [science] thermal-conductivity detector

TCI [drilling] tungston carbide inserts

TCPA [ship] time of closest point of approach

TCP/IP [computers] networking protocol available on the ship

TD [drilling] total depth of a hole

T/D [drilling] top drive

TDK [drilling] tension device (Schlumberger)

TDP [logging] TAM drilling packer (obsolete)

TECP [JOIDES] Tectonics Panel

TEDCOM [JOIDES] Technology and Engineering Development Committee

TGB [drilling] temporary guide base

TIFF [computers] tagged image file format

TIH [drilling] trip into hole (lower drill string below the rig floor, not necessarily below the mud line, by adding drill pipe)

TLT [logging] temperature-logging tool

$T_{\max }$ [science] maximum temperature of hydrocarbon generation during pyrolysis

TOB [ship] thrown overboard (float test failure)

TOC [science] total organic carbon

TOH [drilling] trip out of hole (a.k.a. POOH)

TOTCO drilling rig instrumentation company

TR [ship] transit satellite

TRM [science] thermoremanent magnetization 
TS [drilling] top sub

TSB [curation] thin-section billet (sample designation)

TSINFO [curation] ODP program to track condition and location of ODP thin sections

TSP [logging] Tam straddle packer

TSV [ship] total support vessel

t.u. University of Texas at Austin

TWT [science] two-way traveltime

UBG used but good

UCSD University of California, San Diego

UDI Underseas Drilling, Inc. (now ODL)

UHF ultra-high frequency

UGH [drilling] upper guide horn

UMBRELLA [science] ODP database containing descriptions of all ODP databases

URI University of Rhode Island

USCG United States Coast Guard

USGS United States Geological Survey

USSAC United States Science Advisory Committee

USSSP United States Science Support Program

U.T. University of Texas at Austin

UTC Universal Time Coordinated (used in place of GMT or Z)

UTIG University of Texas Institute for Geophysics

uv ultraviolet

UW University of Washington; U/W, UW [ship] under way (adverb); underway (adjective)

UWG [ship] underway-geophysics laboratory

VAX [computers] Virtual Addressing Extended; the name of the computer used to store ODP data

VCD [ODP] visual core-description form; database program for visual core descriptions of sedimentary cores

VE [science] vertical exaggeration

VHF very high frequency

VIT [drilling] vibration-isolated television (as in "VIT frame")

VLHPC [drilling] variable-length hydraulic piston corer (obsolete)
VMS [computers] Virtual Memory System (operating system of the VAX computer)

VPC [drilling] vibra-percussive corer

VRM [science] viscous remanent magnetization

VSP [logging] vertical seismic profile downhole measurement

WAN [computers] wide-area network

WCR [ODP] West Coast Repository at SIO

WD [ship] water depth

WG [JOIDES] Working Group

WHC [logging] wireline heave compensator

WHOI Woods Hole Oceanographic Institution

W/L, WL [drilling] wireline

WLR [drilling] wireline reentry

$W / L ~ R / E$ [drilling] wireline reentry into a reentry cone without a drill ship

WOB [drilling] weight on bit; waiting on berg (suspended operations in high latitudes)

WOC [drilling] waiting on cement (suspended operations)

WOCE World Ocean Circulation Experiment

WORM [computers] "write once, read many" laser disk drive

WOW [drilling] waiting on weather (suspended operations)

WPAC [JOIDES] Western Pacific Panel

WP-DPG [JOIDES] Western Pacific Detailed Planning Group

WST [logging] well seismic tool (used in VSP experiments)

WSTP [science] downhole water sampler and temperature probe

WLP [drilling] wireline packer

WLS [logging] Wireline Logging Services

XBT [science] expendable bathythermograph

XCB [drilling] extended core barrel

$\mathrm{XCB} / \mathrm{FC}$ [drilling] extended core barrel flow control system

XO [drilling] crossover (connecting pipe)

XRD [science] X-ray diffraction; [curation] sample designation (or XD)

XRF [science] X-ray fluorescence; [curation] sample designation (or XF)

Z "Zulu" or GMT, replaced by UTC 